

\title{
observation of Neoclassical Effects on \\ Neutral Beam Injected Ions in PIT
}

R. Kaita, R. J. Goldston, and J.-P. Bussac

Princeton Univergity, Plasma Physics Iaboratory,

Box 451, Princeton, New Jersey 08544

\begin{abstract}
The horizontally-scanning charge exchange analyzer on the Princeton Large Torus (PLT) was used to measure fast co-moving ions during counter-injection of neutral beams. The large flux of charge exchange neutrals observed under these circumstances exhibited a sharp cutoff as the asalyzer was moved toward more parallel radil of tangency. At a fixed angle and beam energy, the cutoff showed a strong dependence on the plasma current. Monte-carlo calculations using a Fokker-Planck formalism and full toroidal geometry give substantive agreement with the experimental data, indicating that neoclassical effects on neutral beam injected lons were directly obse. 'ed.
\end{abstract}

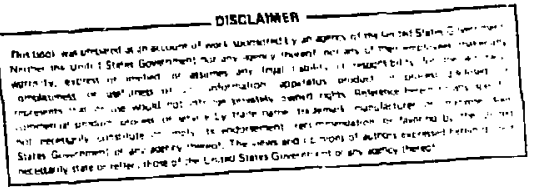


1. Introduction

The study of charge exchange spectra from suprathermal ions is an important means of understancing plasma dynamics in the large magnetic confinement devices now in operation. Ion temperatures are routinely obtained from the distribution of charge exchange neutrals detected by a radially scanning neutral particle analyzer, and a horizontally-scanning analyzer has been used to study the momentum and energy transfer to the plasma from beam tons, by measuring the charge exchange spectra from the thermalizing lons.

More recent1y, there has been interest in a direct means of determining if trapped particles (in banana orbits) behave neoclassically in tokamak plasmas, The Fast Ion Diagnostic Experiment (FIDE, the name given to the horizontally-scanning analyzer) is well suited for making the appropriate measurements. The neutral beams on PLT are oriented to inject tangentially. When beam ions are first reflected by the gradient in the toroidal field as they slow down and scatter in angle, the geometry of the resulting barelytrapped particle orbits are such that the fast neutrals can be viewed over a Iimited range of the horizontal detector scan. Furthermore, due to finite banana-width orbit topology, this range of view for barely-trapped orbits is reduced as the plasma current decreases. Experimentally, substantial co-going neutral flux is observed during counter-injection in the range of angles corresponding to barely-trapped particles. In addition, the angula = range over which the onhanced flux is observed narrows as the plasma current is reduced.

In a preliminary analysis of the data, the distribution of allowed cogoing particles that are barely-txapped in banana orbits was calculated. These rbits depend on the poloidal field, and since this in turn is a function of the plasma current, the dependence of the measured flux on the plasma current 
is uetermined for suprathermal ions in tokamak plasmas. The calculated cutoff angle, and its dependence or the plasma current, is in good agreement with the experimental measurements.

For a more complete atudy of the data, a Monte-carlo computer program was used to simulate the evolution of the beam particle distribution. Each particle was followed as it was launched as a neutral into the plasma at 40 kev. Its orbital motion and slowing down were deterinined by integrating the guiding center orbit equations for axisymmetric systems and by including the effects of a Fokker-Planck collision operator.[1] when the particle slowed down to $30 \mathrm{kev}$, or if it was lost by charge exchange or collision with a limiter, the same orbit-following calculation was performed for $a$ new particle. The expected charge exchange distribution, generated when the process was repeated for a large number of particles, is in general agreement with the experimental data. We thus conclude that the unexpected experimental result of observing comparable co-going and counter-going charge exchange Eluxes at some angles Juring counter-injection of neutral beans in PiT, is consistent with finite banana-width neoclassical theory for suprathermal ions in tokamak plasmas.

\section{Experimental observations}

$40 \mathrm{keV}$ neutral hydrogen beams were injected into an ohrically-heated PLT deuterium plasma. A counter-beam was turned on for about 70 milliseconds, followed after a 30 inillisecond interval by two simultaneous co-beams of the same duration. The Fast Ion Diagnostic Experiment (FIDE) horizontally-scanning neutral particle detector was aimed to look at sightlines with radji of tangency from $72 \mathrm{~cm}$ to $137 \mathrm{~cm}$, at roughly ten $\mathrm{cm}$ intervals. The tangency 
radius is simply the minimum distance between a particular detector sightline and the center of the plasma, as illustrated by the lines normal to each sightline in Fig. 1. The direction of the plasma current and the relative positions of the neutral beam injectors, which determine whether they are injecting in the co- or counter-direction, are also shown. Although the power In each direction was about $250 \mathrm{kw}$, the changing plasma density made comparison between the detected flux from co- and counter-beams uncertain to about a factor of two. However, it was still possible to make the interesting observation that the fluxes of direct and reflected ions at $30 \mathrm{kev}$ were about. the same in magnitude (Fig. 2). "Direct" in this context indicates cotraveling ions injected in the co-direction, while "reflected" ions are those which are injected in the counter-di cection, but observed in the co-direction. Furthermore, there was a sharp cutoff in the reflected flux between tangency radii of $108 \mathrm{~cm}$ and $118 \mathrm{~cm}$ (Fig. 3), the full angular dependence of the measured flux being shown in Figs. $4 \mathrm{a}$ and $4 \mathrm{~b}$. The cutoff in the flux is evident only when the detector is lonking at co-going ions (Fig. 4a), but not when the plasma current is reversed, and counter-moving ions during coinjection (Fig. 4b) are observed.

The plasma current was then varied with only counter-injection. As is evident in Fj.g. 5, the sightline along which fart. ions can no longer be seen becomes progressively more perpendicular as the plasma current is decreased. How complete this cutoff actually is can be seen in Table I, which records the relative magnitude of the fluxes. When the toroldal field was increased from 20 kilogauss to 32 kilogauss, however, there was no effect on the position where this cutoff in olgnal occurred.

All of these phenomena can be explained qualitatively in terms of counter-injected fast ions undergoing pitch-angle scattering into barely- 
trapped banana orbits. Although thelr birth points may occur at falrly 5 mall minor radii, their maximum spatial excursions may be quite large, ilmited only by the dimensiong of the tokamak vacuum vessel. Since the co-branch of the resulting "World's Fattest Banana" (WEB) [2] orbit travels in the high neutral density region near the plasma periphery, a substantial charge exchange efflux is generated in the co-direction. These considerations are included in the calculations used to model the experimental results.

\section{Reflected Flux calculations as}

a Function of the Plasma Current

To see if the plasma current dependence of the cutoff in the fast ion flux coula be predicted, calculations were performed to determine the allowed distribution of reflected ions as a function of detector sightline. The width of the trapped-particle banana ortits is proportiunal to the poloidal field gyroradius, which in turn depends on the plasma current. The orbits whose banana tips nearly touch each other have the maximum widths, and it is the particles in these WFB orpics which are the reflected lons with the largest minor radii. Since the FIDE sightlines are in tl midplane of the plasma, the cosine of the pitch-angle of the ion as it crosses the midplane determines whether or not the particle, once tt leaves the plasma through charge exchange, will be directed toward the detector.

The values of $v_{1 /} / v$ on the plasma midplane for the co-branch of all WFB orbits in PII are plotted as a function of major radius in Fig. 6. From the toroidal geometry of the plasma, the sightlines of the detector in this representation are determined by 


$$
\frac{R_{t}}{R}=\frac{v_{11}}{v}
$$

where $R_{t}$ is the radius of tangency of the sightline, and $R$ is the major radius at which an ion with a particular $v_{1 /} / v$ crosses that sightline. Thus only those ions having the appropriate $v_{\|} / v$ at a given major radius can enter the detector. This inverse dependence on the major radius is depicted in the curvature of the four sightlines also shown in Fig. 6, and their intersection with the curve for the trapped-particle orbits determine at which analyzer tangency radil the reflected ions should be seen. The results are seen to agree vell with experiment.

\section{Fokker-Planck Beam-Orbit Calculations}

A more difficult problem is to calculate the magnitude of the detected flux of reflected ions, and for hhis an orbit-following Monte-Carlo computer program was used. [3] Electron temperature and density profiles from TV Thomson scattering measurements were used as input, and the neutral density profile was calculated using a Monte-Carlo code.[4] This program used the wall of the vacuum vessel as a source of neutrass, and it allowed for recombination within the plasma. The resulting profile (as a sunction of minor radius $r$ ) is depicted in Fig. 7 .

The orbit-following program uses Monte-Carlo techniques to simulate beam deposition witíln the plasma. Fast ion orbits are ther calculated as each beam particle is allowed to alow down, its velocity changing due to the binary, small-angle collisions assumed in the Fokker-planck theory. [5] Ion and electron drag, energy diffusion, and pltch-angle scattering are effects which are included in the code. The calculation continues until the particle 
slows down to $27 \mathrm{kev}$, or is lost as a result of a collision with a limiter or charge exchange transport out of the plasma. Excursions of orbits into the region between the plasma perimeter and the vacuum vessel are allowed, and the limiters were modeled as localized on the torus as thoy actually are on PLT. When the particle's energy is between $29.5 \mathrm{keV}$ anci $30.5 \mathrm{keV}$, it makes a contribution to $f\left(R,\left.v\right|^{/ v)}\right.$ each time it crosses the plasma midplane. Since the detector views only a narrow region about the horizontal midplane, the contribution from each crossing is inversely proportional to the magnitude of $v_{y}$, where $v_{y}$ is the vertical guiding center drift motion. The fast ion distribution calculated in this manner from many orbits is then weighted according to the neutral density profile that increases strongly from the center of the plasma to its edge.

A plot of the weighted distribution function obtained after 10,000 particles were followed is shown in Fig. 8 a for co-injected particles, and in Fig. $8 b$ for counter-injected particles. The curved lines correspond to the sightlines of the FIDE detector. A seconl computer code was used to integrate along these sightlines to determine the flux expected at each angle. Each integration step was weighted by the density of particles having the required $v_{1} / v$ at a given major radius to enter the detector along a specific sightline, and the effect of attenuation of the emerging neutrals as they travel through the plasma was also included.

The data whlch have beon discussed up to this point were taken in 1979, when PLT had a toroidal field ripple of about 2.78 on axis, due to reduced current in one TF coll. Data were also taken in 1978, when there was no ripple, and they do not differ in any significant way from the subsequent year's measurements. The ldentical analysis was performed for these data, and they are essentially the same as those associated with the 1979 data. 
A comparison between the 1978 ard 1979 experimental and calculated results is made in Tables II.A and II.B respectively. In all cases, there is a sharp drop in the reflected ion flux as the detector tangency radius increases. As the sightlines become more nearly perpendicular, the flux also decreases, but much less precipitously. The calculations indicate that there are many more direct ions than reflected ions during cominjection (Fig. Ba), while during counter injection, the reflected flux can be quite substantial (Fig. Bb).

\section{Large Angle Scattering Effects}

Beam ions in passing orbits can become banana-trapped through large
angle scattering (LAS) in a single encounter. While this process is infrequent, it is not treated properly in a Fokker-planck calculation which is based on small angle scattering. Its effect was simulated in the Mon't-rarlo orbit calculation in the following way.

The point at which a particular ton is scattered is determined by evaluating the mean Eree path (MFP) integral

$$
\int^{x} \lambda^{-1} \operatorname{MFP} d x^{\prime}=\ln r
$$

where $r$ is a random number greater than zero but less than or equal to one. The integrand has the form

$$
\begin{aligned}
\lambda_{\text {MFP }}^{-1} & =n_{e} z_{\text {eff }}{ }^{\sigma} \text { LAS }^{v} \\
& =n_{e} z_{\text {eff }} \times 1.6 \times 10^{-14}\left(\frac{1}{(E(k e V)}\right)^{2} v
\end{aligned}
$$

where $\sigma_{\text {LAS }}$ is simply the coulomb cross section for 90-degree scattering. The 
plasma impurity factor $z_{e f f}$ and the eleciron density $n_{e}$ used in our calculations were determined from TV Thomson scattering laser scans.

When large angle scattering occurs, the parallel velocity of the ion is modified, and the geometry governing the new parallel ion velocity (v|l) is shown in Fig. 9 .

By definition,

$$
\begin{aligned}
& v_{||}=\frac{\vec{v} \cdot \vec{B}}{|\vec{B}|} \\
& =\frac{1}{B}\left(v^{\prime} \sin (\Delta \theta) \cos \phi^{\prime} B \sin \beta \cos \alpha\right. \\
& +v^{\prime} \sin (\Delta \theta) \sin \phi^{\prime} B \sin \beta \sin \alpha \\
& \left.+v^{\prime} \cos (\Delta \theta) \mathrm{B} \cos \beta\right) \\
& =v^{\prime}\left(\sin (\Delta \theta) \sin \beta \cos \left(\phi^{\prime}-\alpha\right)+\cos (\Delta \theta) \cos \beta\right) \\
& x^{\prime}=\frac{v_{1} I^{\prime}}{v} .
\end{aligned}
$$

$\Delta \theta$ is calculated from the Rutherford scattering formula, based on an impact parameter, b, randomly chosen over the disk within the 90-degree scattering radius. Then by using a random number generator to provide $\phi^{\prime} x^{\prime}$ is obtained, and this new pitch-angle variable is subsequently used in the ion orbit calculation.

The results for calculations with and without IAS, using the beam and plasma parameters associated with the 1978 data, are listed in Table III. From them, it appears that LAS does not contribute signiflcantly to the reflected ion flux. 


\section{Results and Conclusions}

Precise comparison of the experimental and calculated results is made somewhat problematical by a number of effects. First, the density rose during the course of the discharges while the 1979 data were being taken. The direct fast ion signal is observed to vary as the inverse square of the line average plasma density, so that the observed doubling of the density between the counter and subsequent co-injection pulses leads to a best guess for the actual ratio of reflected to direct ions of about 258 of the value which seems apparent from the raw data. This correction has been made in the values listed in Table II.B.

A second important consideration is that the charge-exchange production rate depends on the neutral density profile used. Its exact shape beyond the plasma periphery, where the density is calculated to rise by approximately an order of magnitude, is not well known. Since the radial extent of the wFB orbits allows ions to pass through this region, uncertainties in the neutral profile there can strongly affect the magnitude of the calculated fast ion Elux.

Finally, the precise q-profile is important for determining the angle at which reflected ions are no longer detected. We have used a q-profile of the זัorm

$$
q(r)=\frac{B_{T^{I}}}{B_{p}(a) R_{o}}\left\{\frac{1-\left(1-(r / a)^{2}\right)^{q} r a t}{r / a}\right\}
$$

which results from assuming that

$$
J \propto\left(1-(r / a)^{2}\right){ }^{\left(q_{\text {rat }}-1\right)} .
$$


$B_{t}$ and $B_{p}$ are the toroidal and poloidal magnetic fields respectively, and $q_{r a t}$ is the ratio of $q$ at the edge of the plasma $[q(a)]$ to $q$ on its axis $[q(0)]$. For the varying current cases, the assumed $q(0)$ is shown in $F \pm g$. 6 whtle for the 1979 and 1978 Monte-Carlo analyses, we set q(0) equal to unity.

In light of these uncertainties, cautton must be exercised when examining the precise details of the comparison between observation and theory. However, the relatively large size of the reflected flux in both observation and theory, and the substantive agreement between the calculations and the data, clearly indicate that neoclassical effects on fast ions, consistent with finite banana-width theory, are being jirectly observed on PLT with the FIDE technique.

Acknowledgments

Work supported by the $U$. 5 Department of Energy Contract No. DE-ACO2-76-CHO-3073. 
References

[1] J. G. Cordey, Nuclear Susion 16 (1976) 439.

[2] T. H. Stix, Plasma Physice 14 (1972) 367.

[3] R. J. Goldston, Ph. D. Thesis, Princeton University, 1976 (unpublished).

[4] H. H. Towner and R. J. Goldgton, Bull. Am. Rhys. Soc. 24 (1979) 1107.

[5] M. N. Rosenbluth, W. M. MacDonald, and D. L. Judd, Physical Review 107 (1957) 1. 
Figure Captions

1. Relative positions of the neutral beim Injectors and the detector of the Fast Ion Diagnostic Experiment on PLT.

2. Flux of fast ions along four detector sightlines. The first signal shows the flux of reflected counter-infected lons, and the second shows the same for direct co-injected ions.

3. Fast ion flux along four detector sightlines, with the sharp cutoff in the flux of reflected counter-injected ions evident between $108 \mathrm{~cm}$ and $118 \mathrm{~cm}$.

4. Angular distributions of the measurea fast ion fluxes for (a) co-moving ions during counter-injection, and (b) counter-moving ions during coinjection. The range of tangency radii around $120 \mathrm{~cm}$ in each graph indicates regions in which the data were cunsistent with zero.

5. Measured fast ion flux as a function of plasma current. The sightline along which no flux is seen becomes successively more perpendicular as the plasma current is decreased. The tangency radii of the four channels and the magnitudes of the fluxes seen in each of them are listed in Table $I$.

6. $v_{\|} / v$ as a function of major radius for the co-branch of all wFB orbits in PLT. The sightlines are labeled according to the corresponding channels in Fig. 5.

7. Calculated neutral density as a function of minor radius.

8. Plot of weighted distribution function $n_{0} f\left(v /\left.\right|^{/ v, R}\right)$, obtained from Monte-Carlo calculations, for (a) co-injected beam ions, and (b) counter-infected beam lons. The uppermost sightline in Fig. $8 b$ is the one along which reflected fast ions are no longer detected. 
9. Coordinate system showing change in parallel ion velocity when an ion undergoes large angle scattering. 
TABLE I

Reflected Fast Ion Flux Dependence on the Plasma current

Charge Exchange Flux Level

(Arbitrary Units)

\section{Plasma}

Current $(\mathrm{kA}$

Channel

$\left(R_{\tan }=87 \mathrm{~cm}\right)$

$5 \pm 1$

$8 \pm 2$

$10 \pm 1$

$3 \pm 1$

$13 \pm 3$

$22 \pm 2$
Channel 3

$\left(R_{\tan }=107 \mathrm{~cm}\right)$

$9 \pm 1$

$<1$

$<1$

1
Channel 4

$\left(R_{\tan }=11: \operatorname{sm}\right.$

100

$\left(\mathrm{R}_{\tan }=97 \mathrm{~cm}\right)$

$<1$

$<1$

150

$\div 9$ 
TABLE II . A

COMPARISON OF THE 1978 FAST ION DIAGNOSTIC

DATA WITH CALCULATED RESULTS

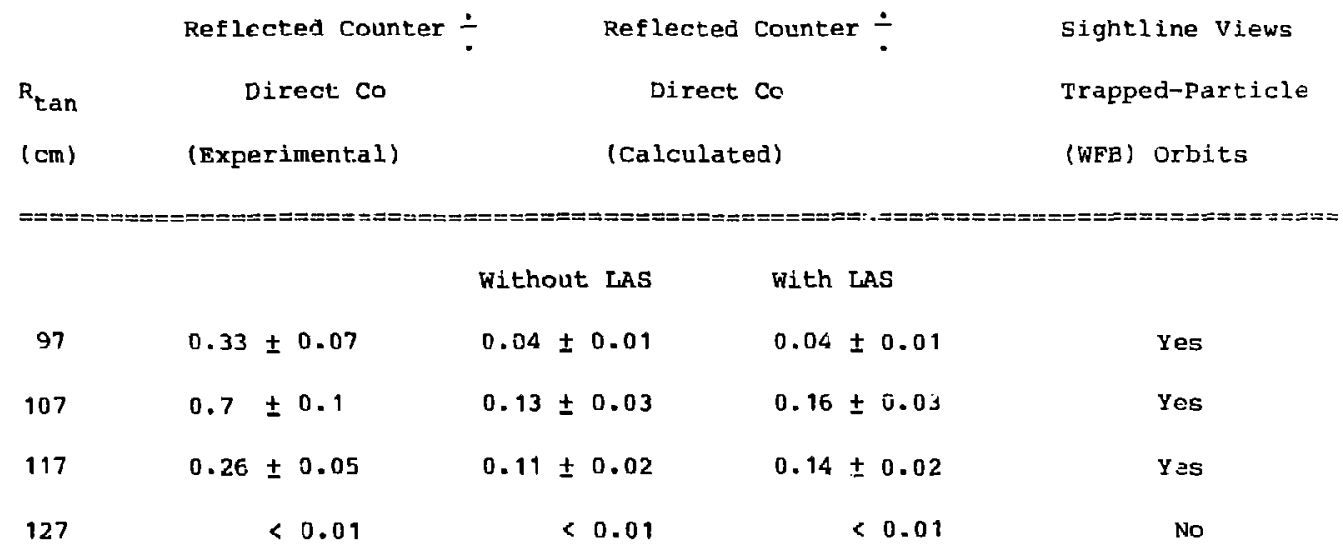


Table II. B

RATIO OF RELFECTED TONS TO DIRECT IONS FOR THE 1979 DATA

\begin{tabular}{|c|c|c|c|c|c|c|}
\hline$R_{\text {tan }}$ & \multicolumn{4}{|c|}{ Experimental } & \multicolumn{2}{|c|}{ Caiculated } \\
\hline$(\mathrm{cm})$ & Count & er-Inj. & Co-Inj & & Counter-Inj. & Co-Inj. \\
\hline 93 & 0.38 & \pm 0.08 & 0.017 & \pm 0.003 & 0.12 & 0.016 \\
\hline 102 & 0.28 & \pm 0.06 & 0.013 & \pm 0.003 & 0.08 & 0.015 \\
\hline 108 & 0.33 & \pm 0.07 & 0.011 & \pm 0.002 & 0.14 & $<0.01$ \\
\hline 118 & & $<0.01$ & & $<0.01$ & 0.11 & $<0.01$ \\
\hline 127 & & $c 0.01$ & & $<0.01$ & $<0.01$ & $<0.01$ \\
\hline
\end{tabular}




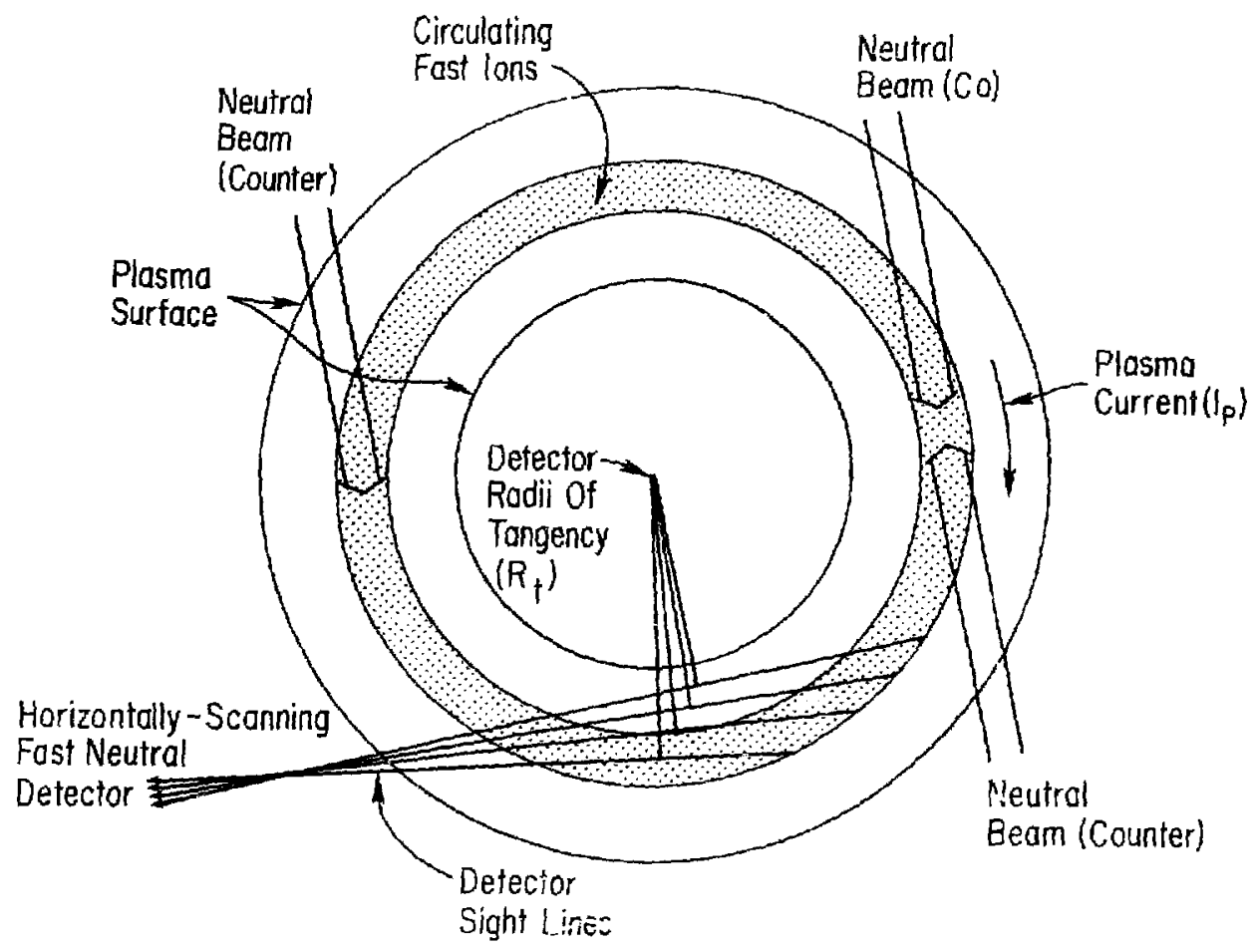

Fiq. 1. (PFPL-806868) 

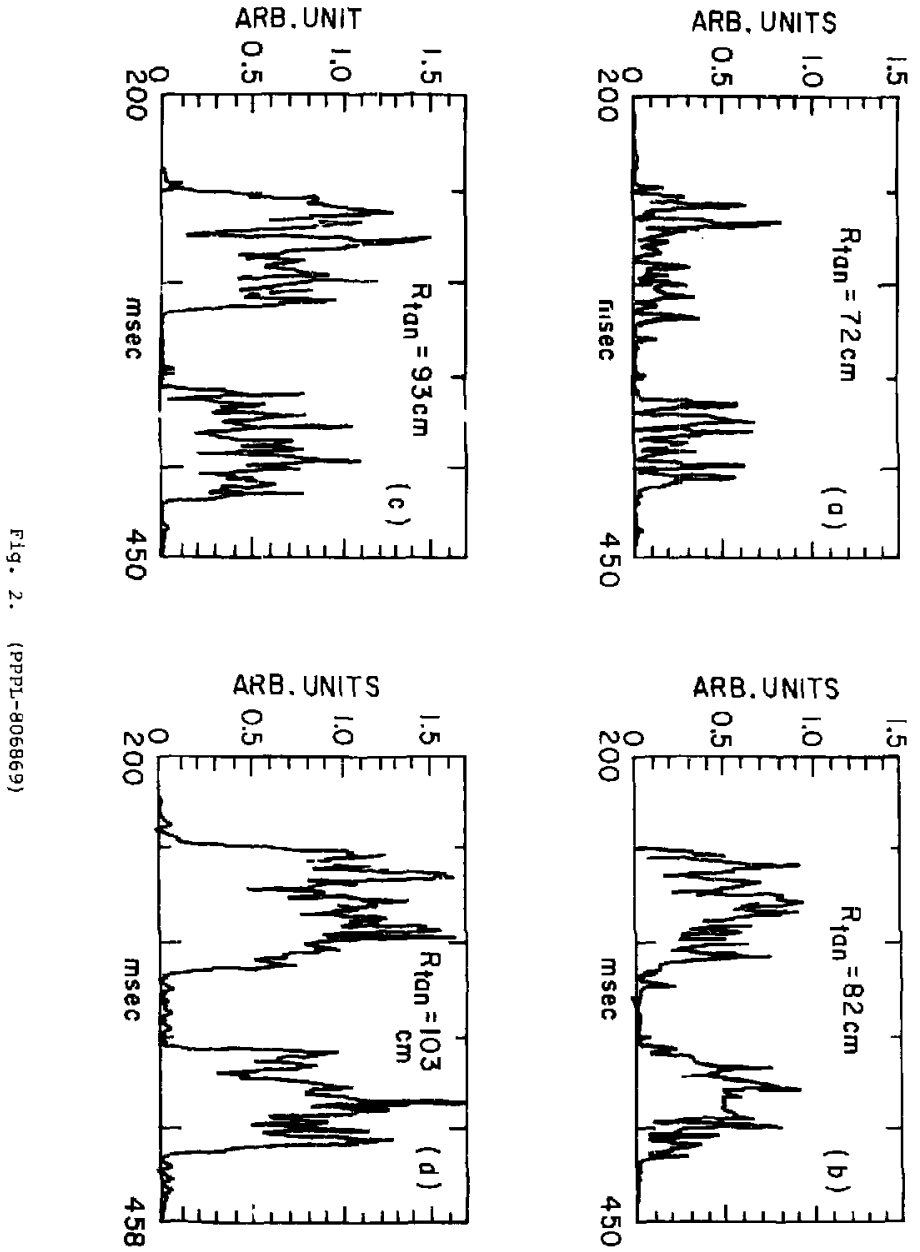

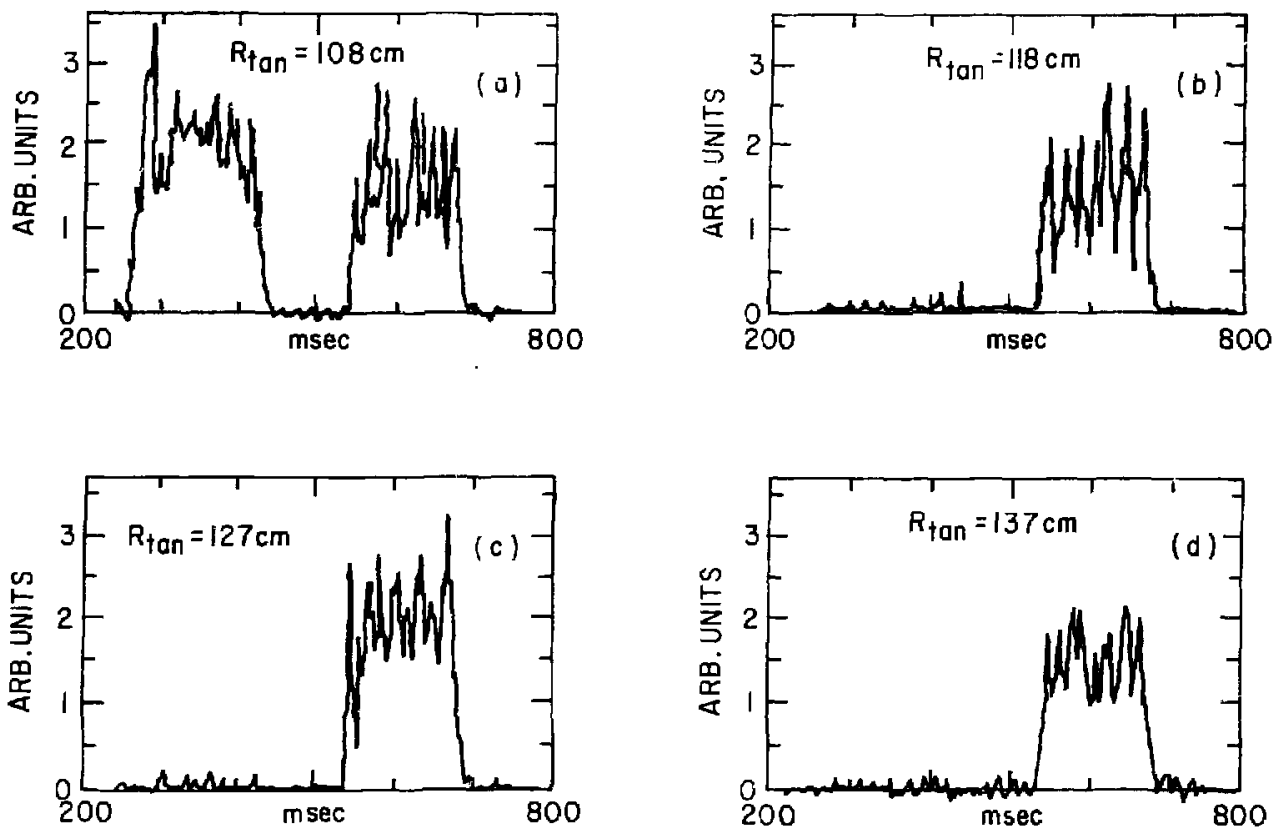

Fig. 3. (PFPL-806873) 

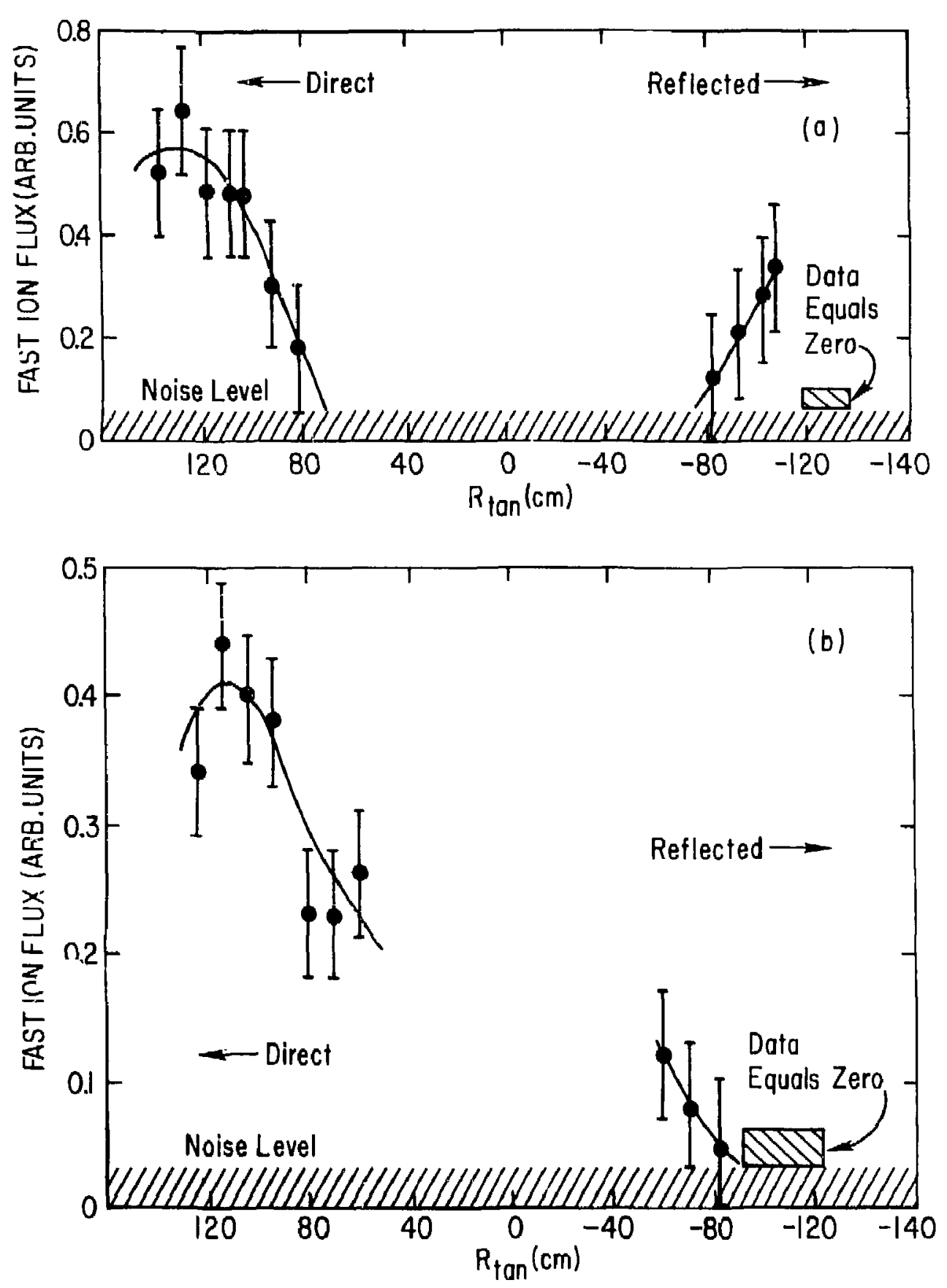

Fig. 4. (PPPL-906874) 


$$
i_{p}=290 \mathrm{kA}
$$
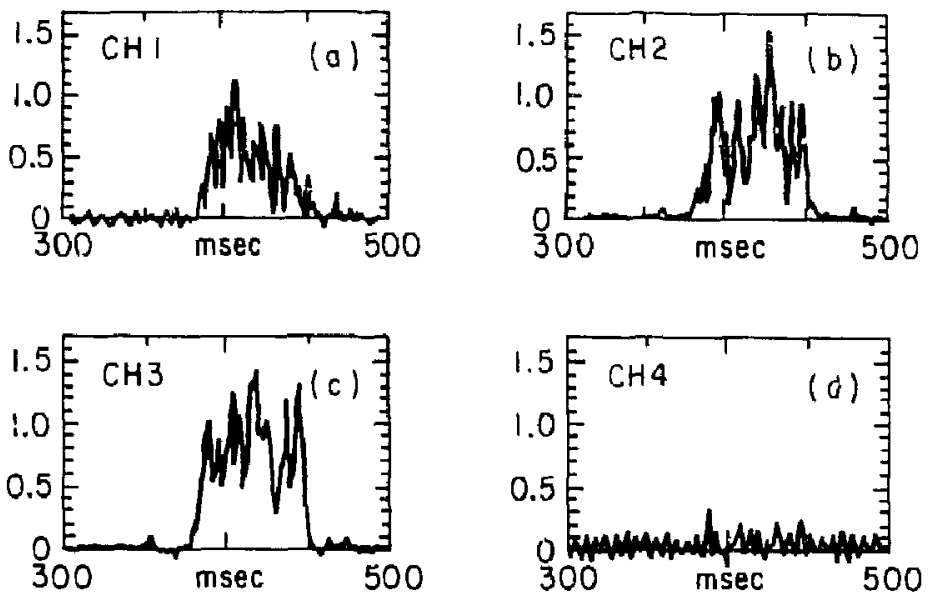

$I_{p}=150 \mathrm{kA}$
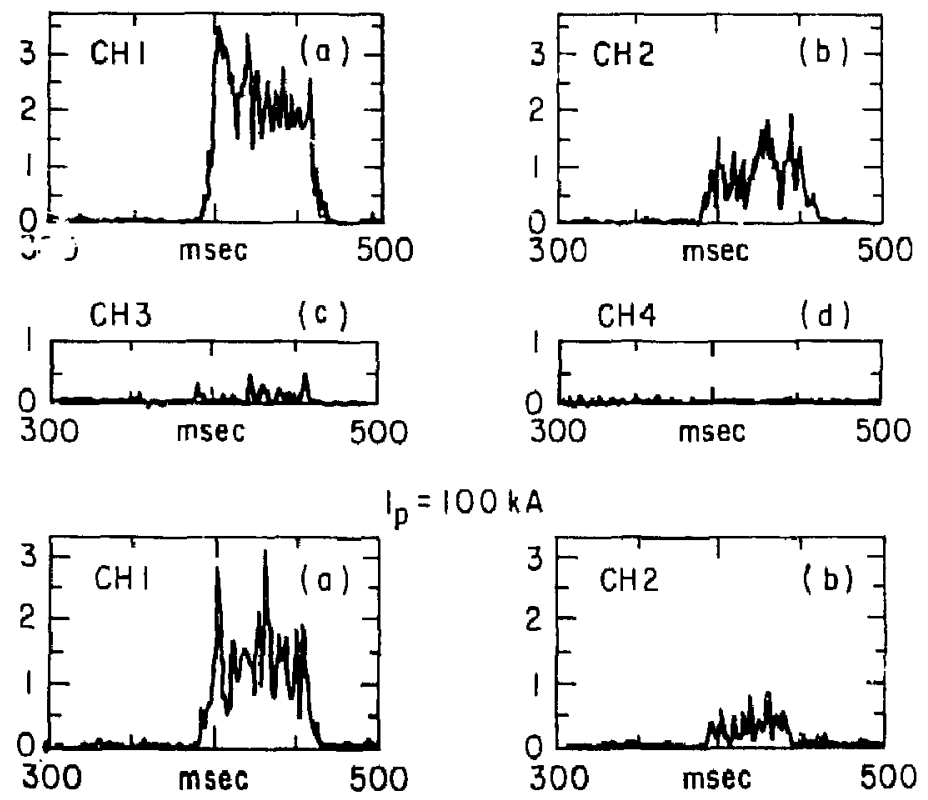

$I_{p}=100 \mathrm{kA}$
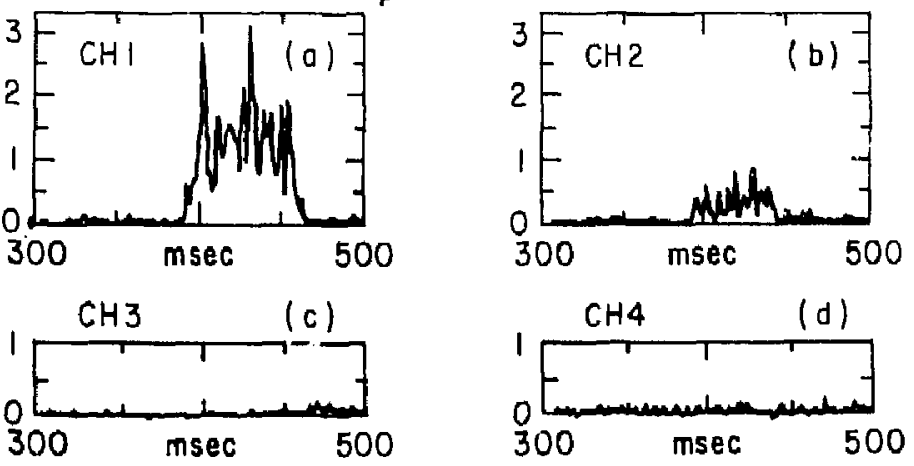

Fig. 5. (PPPL-806871) 

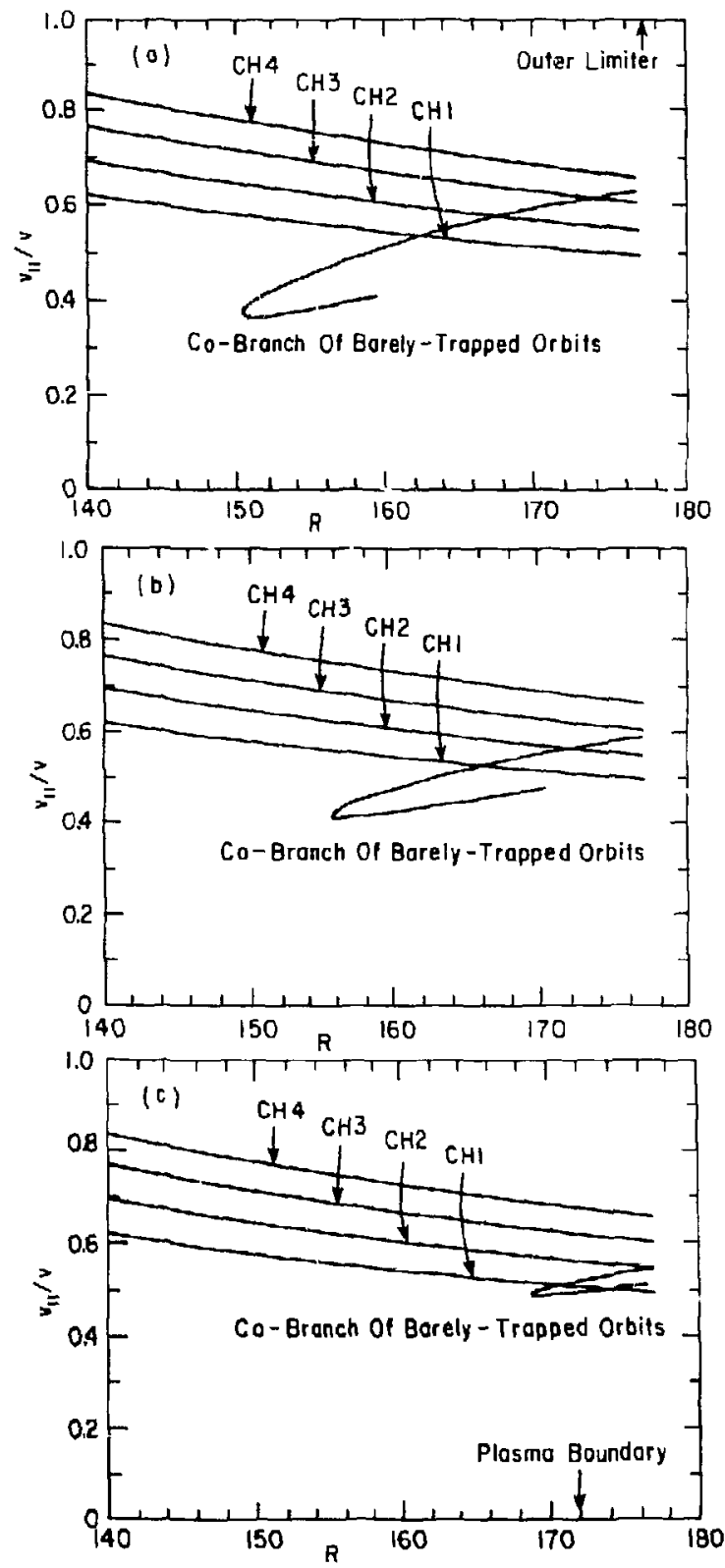

Fig. 6. (PPPL-806870) 


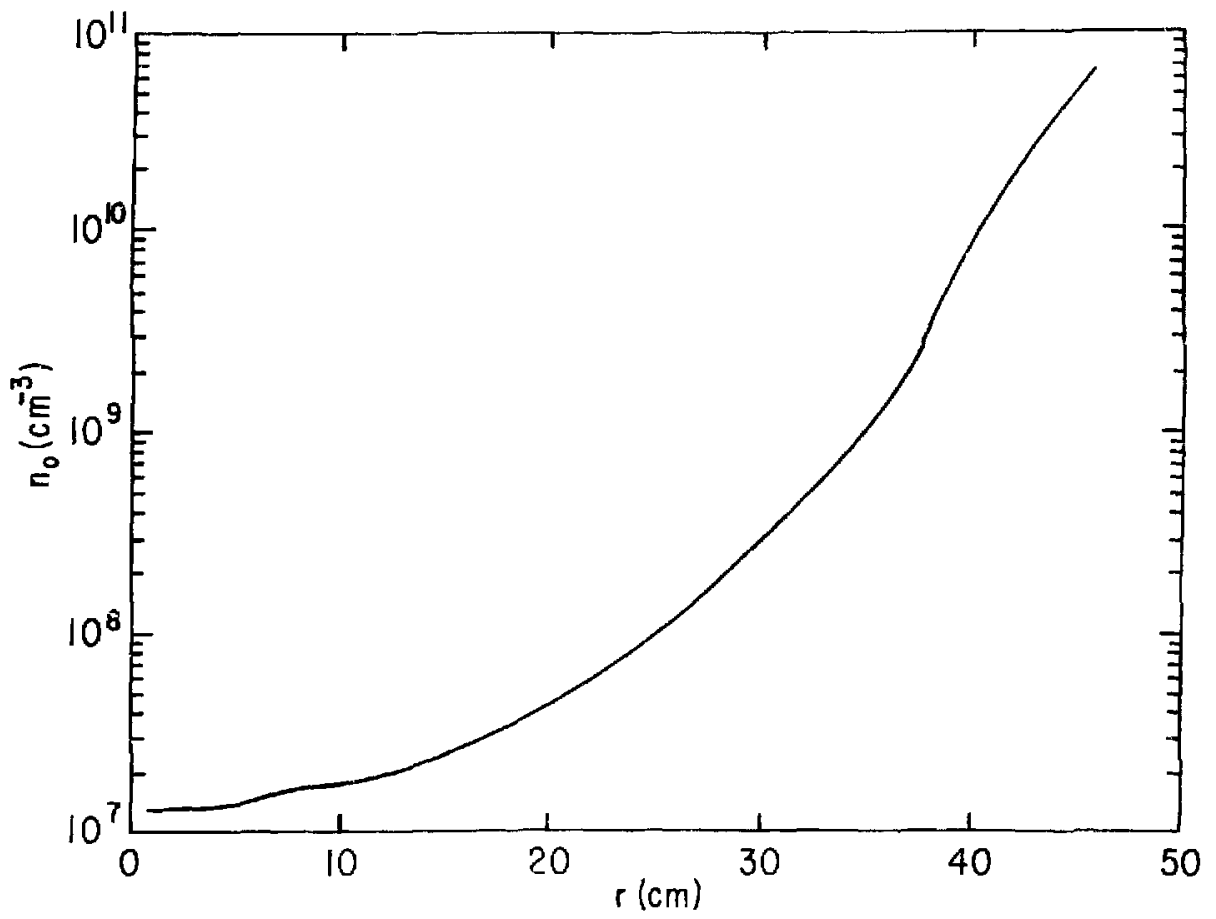

Fí 7. (PFPL-806866) 



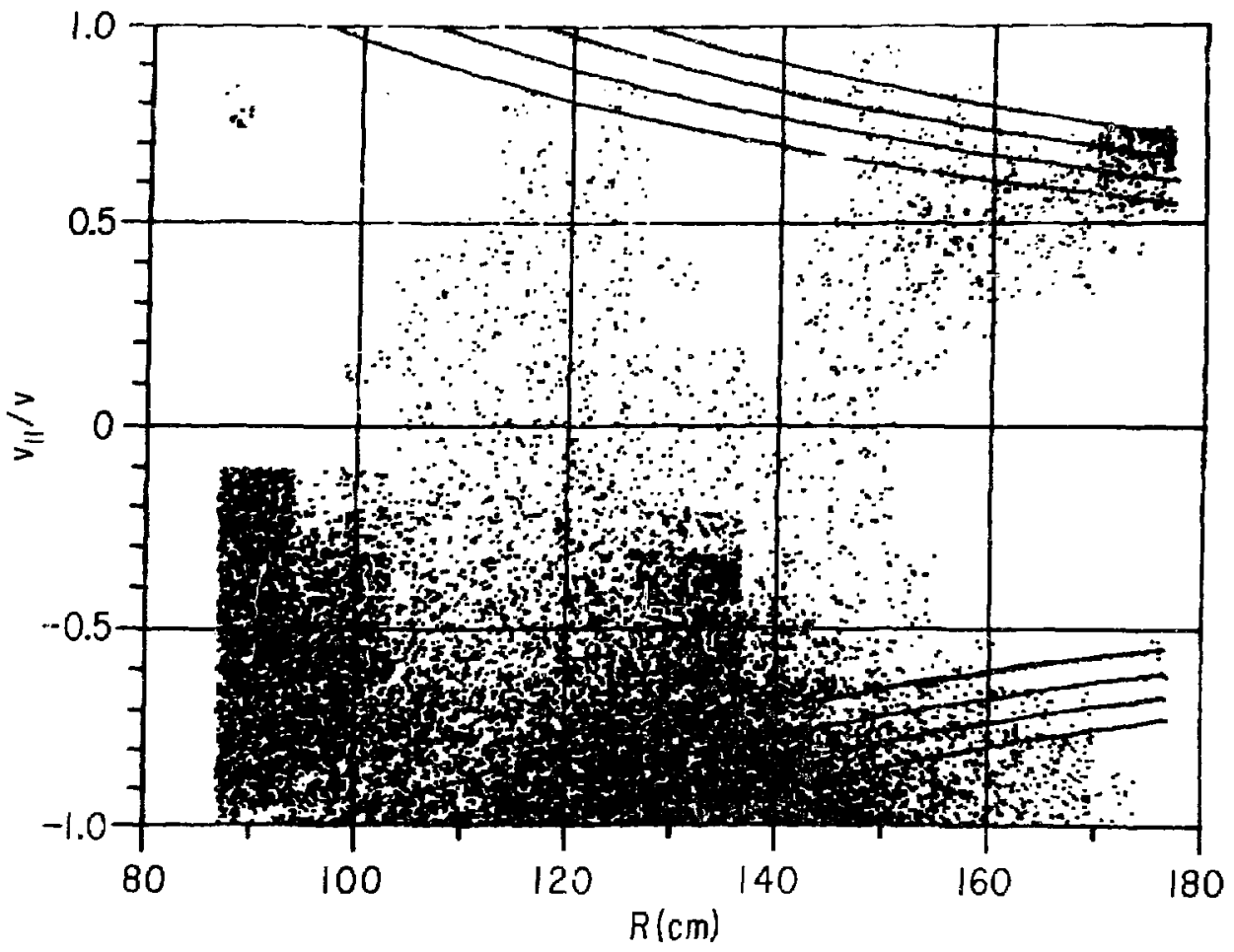




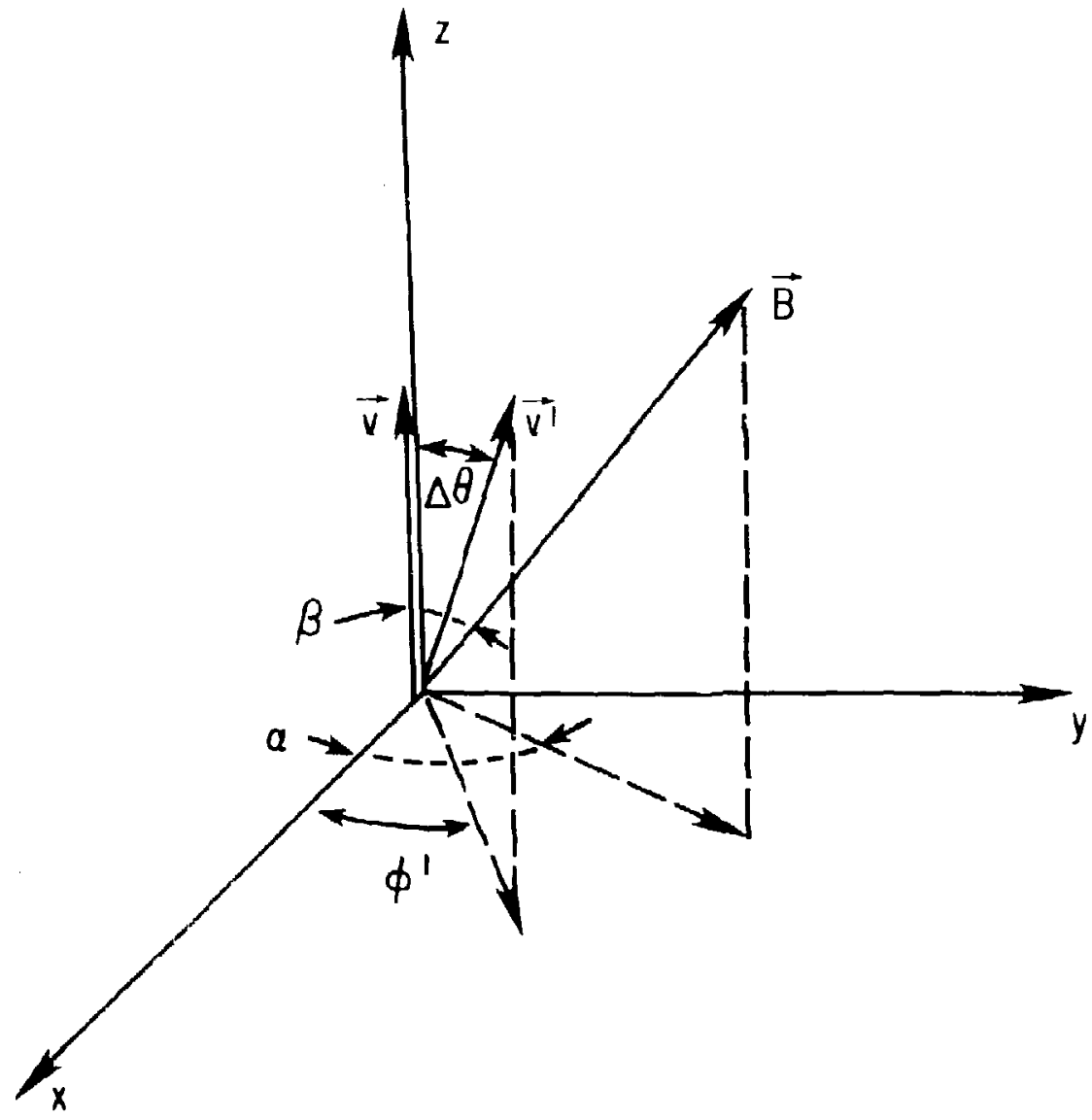

Fi.g. 9. (PPPL-806872) 